\title{
Design and Analysis of a Soft Pneumatic Actuator with Origami Shell Reinforcement
}

\author{
Laura Paez, ${ }^{*}$ Gunjan Agarwal, ${ }^{*}$ and Jamie Paik
}

\begin{abstract}
Soft pneumatic actuators (SPAs) are versatile robotic components enabling diverse and complex soft robot hardware design. However, due to inherent material characteristics exhibited by their primary constitutive material, silicone rubber, they often lack robustness and repeatability in performance. In this article, we present a novel SPA-based bending module design with shell reinforcement. The bidirectional soft actuator presented here is enveloped in a Yoshimura patterned origami shell, which acts as an additional protection layer covering the SPA while providing specific bending resilience throughout the actuator's range of motion. Mechanical tests are performed to characterize several shell folding patterns and their effect on the actuator performance. Details on design decisions and experimental results using the SPA with origami shell modules and performance analysis are presented; the performance of the bending module is significantly enhanced when reinforcement is provided by the shell. With the aid of the shell, the bending module is capable of sustaining higher inflation pressures, delivering larger blocked torques, and generating the targeted motion trajectory.
\end{abstract}

Keywords: soft robots, soft pneumatic actuators, origami shell, bending module, elastomers, reconfigurability

\section{Introduction}

$\mathbf{S}^{\mathrm{o}}$ OFT ROBOTS ARE CHARACTERIZED by their light weight, compliance, flexible design choices ${ }^{1-4}$ and embeddability within the structure of a robot. ${ }^{5-8}$ A commonly used actuator in soft robotics is the soft pneumatic actuator (SPA), which is actuated by the use of compressed air. $^{9-18}$ In particular, bending SPAs derive their deflection from a straight to bent configuration due to the air pressure built within their chambers. This bending motion is suitable for a diverse set of applications such as gripping and manipulating fragile objects, ${ }^{9,17}$ adaptable locomotion on unstructured terrains and autonomous navigation, ${ }^{19,20}$ operating surgical tools, ${ }^{21}$ and biomedical rehabilitation. ${ }^{22,23}$ The elastomeric materials used to fabricate SPAs typically present high resilience and robustness to different contact environments. ${ }^{10}$ However, once inflated with pressurized air, SPAs suffer from some inherent disadvantages such as the lack of precision in movement and vulnerability to rupture in their walls. Once activated, SPAs inflate their internal chambers, leading to an overall reduction in the thickness of their walls. If these inflated thin soft wall layers come into contact with any rough particles or surfaces present in the working environment, they are likely to be perforated, damaging the entire actuator. Thus, despite the resilience in material used, $8,12,13$ robots fabricated with soft materials are highly susceptible to punctures and other mechanical failures when inflated under harsh conditions, ${ }^{10}$ or when subjected to large deformations. Consequently, an important requirement for the design of a soft bending module is a protective layer to cover the SPA while allowing for adequate mobility within the actuation range.

To circumvent these issues faced by SPAs upon inflation, some solutions have been proposed to constrain the radial expansion of the SPAs and guide their motion along a predefined desired trajectory. Deriving inspiration from the classical McKibben-style actuator designs, ${ }^{24,25}$ where the air chamber of the actuator is covered with fiber winding. Fiber reinforcements have also been introduced in more recent soft actuator prototypes, ${ }^{26-29}$ where a high-strength fiber material is manually wound on the circumference of the actuator. In some such prototypes, grooves created on the outer surface of the actuator during the fabrication process ${ }^{28}$ guide the placement of outer fiber reinforcement in a predefined pattern, allowing for repeatability in manufacturing. Other methods to constrain the expansion of SPAs involve having a thin outer casing shell of a higher stiffness material attached 
onto the actuator surface, with evenly spaced openings in the shell to allow restricted inflation of the contained actuator. ${ }^{29}$

This work presents a new type of soft bending module in an effort to circumvent some of the above-mentioned drawbacks faced by conventional SPAs and to enable more robust and enhanced performance characteristics. The bending module presented here comprises a bending SPA core, to provide the necessary actuation motion, and a light-weight origami shell structure that guides the motion of the actuator within the desired range of application. The origami shell serves multiple purposes targeted toward achievement of desired displacement and force requirements, by enhancing the mechanical performance obtained with the SPA alone. The use of an origami shell attachment helps combat the inherent issues of a soft actuator such as vulnerability in the SPA walls and incapability to withstand high levels of pressure without damage to the actuator. It provides mechanical guidance for actuation motion and constrains the maximum displacement as well as inflation limits for the SPA. With assistance from the shell, the bending module is also able to deliver higher forces and torques desirable in several applications such as gripping, for instance. Furthermore, the origami shells proposed here are made from light-weight, low-cost, and easily available materials such as paper and have predefined periodic patterns fabricated on them for guided motion assistance, in an effort to make the entire fabrication process more manufacturable and repeatable. The origami pattern used for the paper shell dictates the mechanical properties of the entire structure, such as the stiffness and the resilience. By changing the pattern of the origami shell, it is possible to tune the mechanical behavior of the entire bending module and achieve greater stability as well as flexibility in actuation motion. Thus, the soft bending module presented here holds the capability to configure more sophisticated and complex soft actuator-based systems in the future, than those built using soft actuators alone.

Origami, the Japanese art of paper folding, is a technique that allows the generation of rigid folding structures by using a sheet of paper or more recently using thicker materials. ${ }^{30,31}$ Recent work has shown that origami tubes made from thin sheets can be used to create a wide variety of deployable, reconfigurable, and mechanistically unique three dimensional structures. ${ }^{32}$ Some examples of robotic structures deriving origami inspiration can be found in Onal et al., Lee et al., Peraza-Hernandez et al., and Paez et al. ${ }^{33-36}$ Taking into account the structural stability, some common crease patterns in origami are Miura-Ori pattern, Yoshimura pattern, and diagonal pattern. ${ }^{37}$ In this work, the Yoshimura pattern is chosen to create the shell for the bending module as it supports the translational motion desired. The diagonal pattern supports rotary motion while the Miura-Ori pattern supports contraction and expansion in all directions, ${ }^{38}$ not desired for the demonstration here. When the origami structure is capable of delivering motion itself through embedded mechanisms, it is known as active origami. ${ }^{33,39}$ In the present work, the shell structure made with origami techniques does not provide motion by itself; the motion is derived from the SPA. Schematic diagrams of the proposed bending module before and during operation are shown in Figure 1a, b, respectively. The procedure for the attachment of the soft actuator to the origami shell to assemble the complete bending module is shown in Figure 1c. An image of the fabricated module is shown in Figure 1d.

In the following sections, analysis related to the choice of best design for the shell as well as the SPA is described. Subsequently, analysis and testing for the integrated module comprising both the shell and the actuator are shown in context of the performance achieved, describing several characteristic curves extracted by rigorous experimental analysis. Finally, a discussion of the results is provided.

\section{Performance Requirements for Bending Module}

The design requirements of the soft bending module proposed here are based on the servomotor-powered rigid bending modules. This approach opens the possibility to use the proposed module to replicate, on lower load $(<10 \mathrm{~N})$ and speed $(<5 \mathrm{~Hz})$ scales, the applications performed with conventional rigid rotational actuators while offering the
FIG. 1. The soft bending module comprises an origamibased folded paper shell to guide the motion of the soft pneumatic actuator (SPA) contained inside that provides the actual motion. The use of a shell surrounding the actuator prevents the actuator walls from rupture and also constrains the inflation limits of the actuator. Schematic diagrams of the soft bending module before and during operation are shown in (a, b), respectively. A schematic diagram of the procedure for attachment of the soft actuator to the origami shell to assemble the complete module is shown in (c). An actual image of the module is seen in (d). Color images available online at www.liebertpub.com/soro

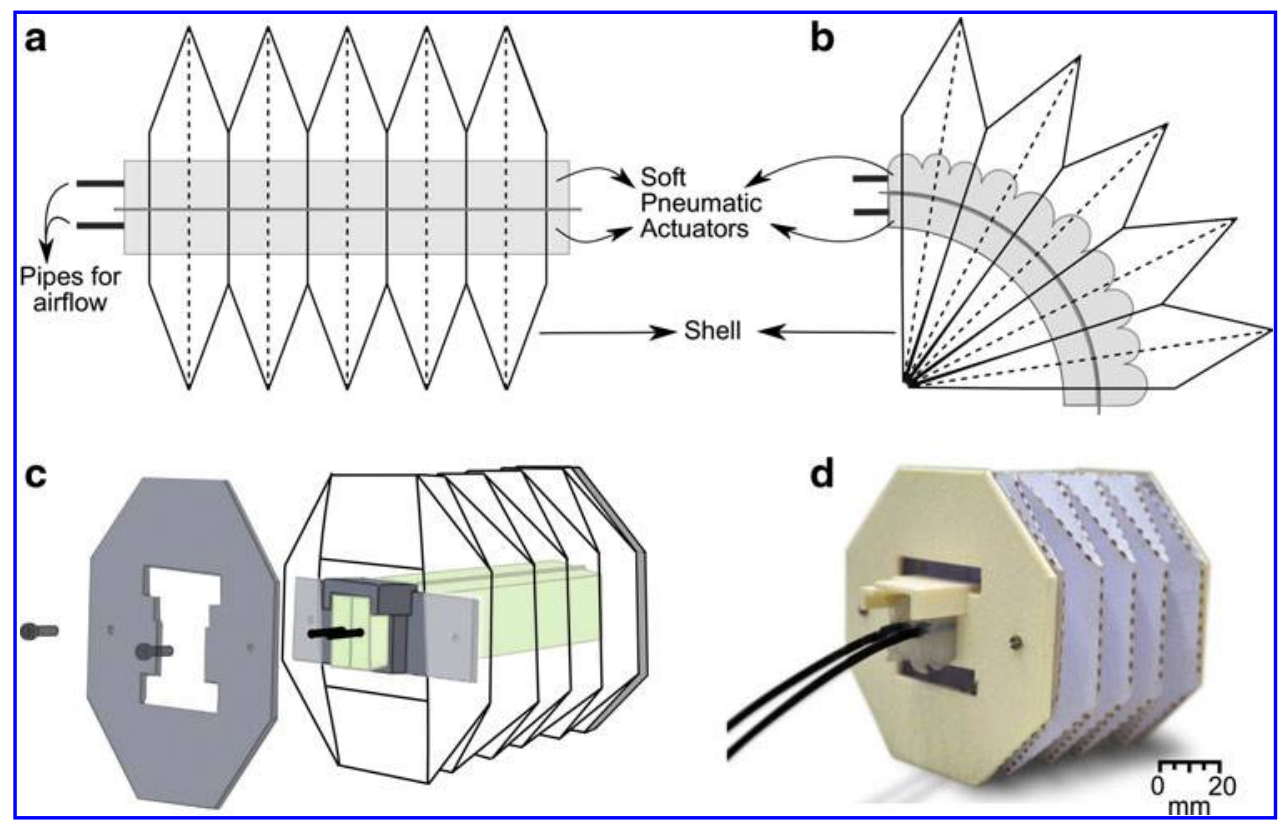




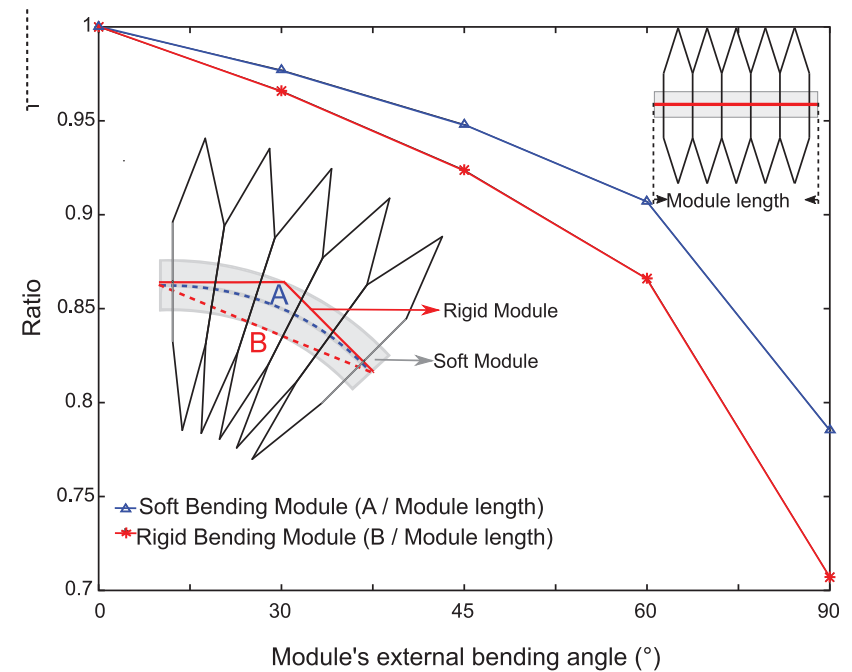

FIG. 2. Comparison of bending characteristics obtained with soft bending modules and rigid bending modules. Ratios of central axis arc length, A, to the total module length are plotted as a function of the bending angle for the soft bending module, and the corresponding ratios of the segment length, B (distance between the module endpoints), to the total module length are plotted for a rigid module. The difference between the bending characteristics obtained with two modules increases with the increase in the bending angle. Color images available online at www.liebertpub.com/soro

inherent advantages of working with soft actuators with their design versatility.

Soft and rigid modules exhibit different bending characteristics. While soft bending modules ascribe an arc shape in the bent configuration, their rigid counterparts, composed of a servomotor and a pair of links, ascribe straight lines. To prove that both rigid and soft modules match the bending expectations with negligible difference, the comparison in Figure 2 shows that there is low difference in the ratios between the central axis arc length to the total module length for a soft module and segment length (distance between the module endpoints) to the total module length for a corresponding rigid module. A maximum discrepancy of $7.8 \%$ is observed between the ratios at the maximum bending angle of $90^{\circ}$, confirming that within the bending range considered, a bending module can closely match a rigid one in terms of kinematics.

At some operating points in the actuation range, the SPAs undergo unconstrained inflation even at constant pressure. ${ }^{11,40}$ This is undesirable if the purpose of the bending module is to bend only a finite angle and maintain adequate strength. In this scenario, the folded paper shell serves as a retainer that provides a restrictive force to the bending actuator. Once all forces (i.e., internal by SPA and external by shell compliance) are balanced, it is expected that the bending module maintains a stable bending condition (i.e., bending angle). This analysis is shown later in the Complete SPA-Origami Module Assembly and Experimental Results section.

\section{Design and Fabrication of Origami Shell}

The foldable origami shell allows for the bending of the SPA internal actuator while simultaneously providing a restoring force that limits the actuator bending to a desirable value. To assemble the module, both ends of the origami shell are designed to have an opening matching the outer diameter of the SPA to be contained. The SPA, along with the tubing attachments for input air supply, is inserted into the origami shell through the opening in the shell designated for the air inlet. Two rigid end attachment plates are then used to clamp the actuator and the shell components of the bending module together in place with the help of screws. Fixtures connected to the inlet end plate are then used to mount and secure the tubing for airflow in place. The proposed origami creates a closed shell that supports translational motion. The use of origami allows for the creation of supporting shells in a variety of geometric patterns, the effect of which on the bending motion can then be characterized. In the following sections, a systematic analysis of the desired structures is presented, leading to the design of the most appropriate fold pattern, along with size requirements and the paper thickness choices.

\section{Origami folding pattern design}

Two Yoshimura patterns were tested as a part of the design process. The major constraint for the design is that the shape of the shell must remain symmetric on the bending plane and along the axis perpendicular to the bending plane. Together, these requirements lead to the design seen in Figure 3. The shell shown in Figure $3 \mathrm{~b}$ uses twice the folding tip length than that used in the shell shown in Figure $3 a$.

As mentioned in the design requirements, there are two functional design criteria that enable selection of the optimal shell pattern. One is the internal volume of the shell, which needs to be large enough to allow the actuator to inflate, but not as to allow the actuator to over inflate. The second criterion is regarding the stiffness of the overall construction. The shell should be stiff enough to restrict any unconstrained overinflation of the actuator, but not too stiff to overconstrain the SPA. To study the effect of both of these design factors on the bending performance, two different configurations for the shell offering different internal volumes and stiffness are studied and characterized, as shown in Figure 3. The pattern in Figure $3 b$ uses twice of the length at each folded tip than the pattern in Figure 3a; therefore, it features a smaller inner volume and more compliance. Both of these shell patterns were analyzed to measure their resistance to deflection or elongation, including the force needed to achieve a constant deflection in both patterns, and the compliance measured in the resulting shapes.

Deflection characteristic. Approximating the origamifolded shell structure edges shown in Figure 3 as a wide beam, ${ }^{41}$ the schematic drawings in Figure 4 illustrate the folded shell edges in Figure 3 subjected to loading. Figure 4a shows a schematic representation of the shell edge for the pattern shown in Figure 3a, and Figure 4b shows a schematic representation of the shell edge for the pattern shown in Figure $3 \mathrm{~b}$. The deflection of the tip of the shell edge can then be expressed by the relation:

$$
\delta=\frac{4 F}{E} \frac{(\text { Length })^{3}}{(\text { Width }) \times(\text { Height })^{3}},
$$

where $F$ is the bending force and $E$ is the paper's Young's modulus. Furthermore, $F_{a}$ is the force applied to bend the 
FIG. 3. Two different origami shells made with Yoshimura patterns. Both shells have similar external volume, with the same maximum height, maximum width, and total length. The two shells differ in the size of the folded tip; the shell shown in (b) uses twice the folded tip length compared to the one shown in (a). Color images available online at www.liebertpub.com/soro

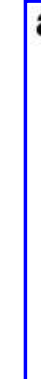

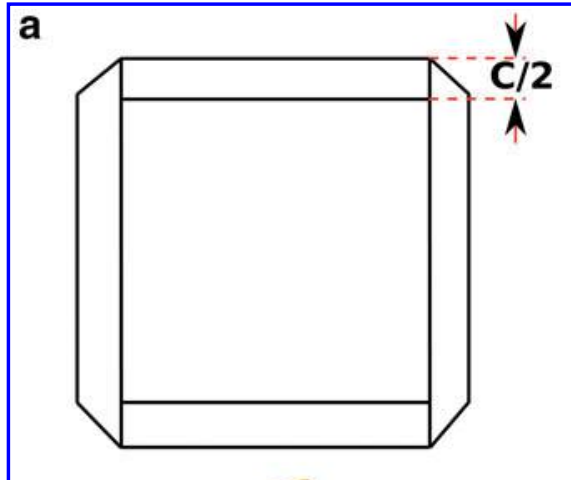

b

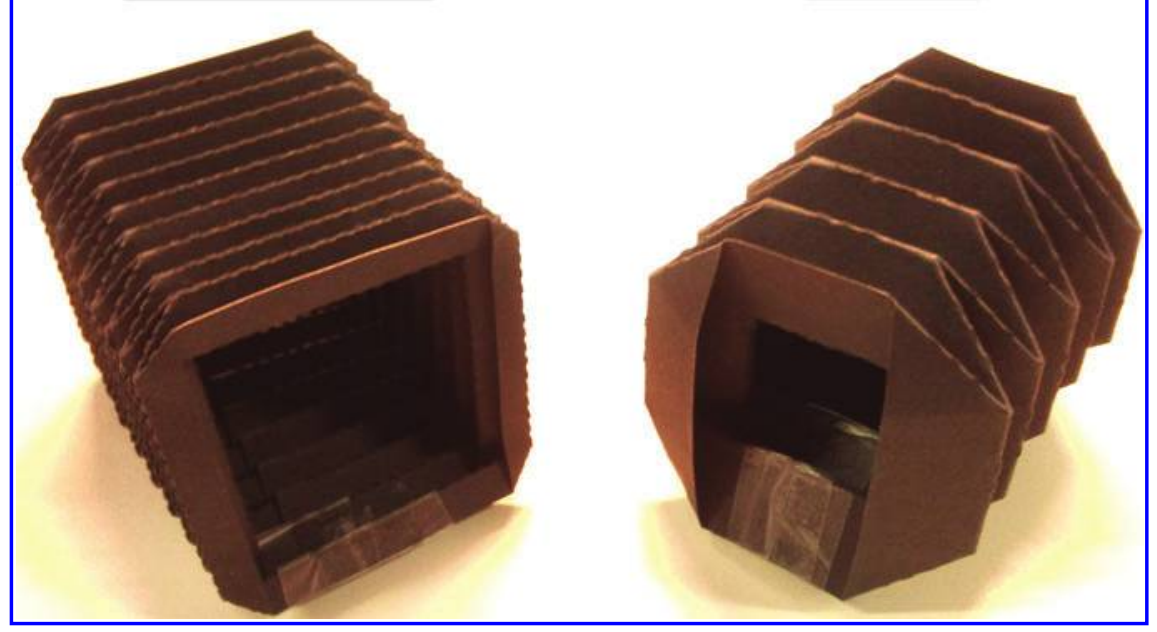

folded edge structure shown in Figure $4 \mathrm{a}$ and $F_{b}$ is the corresponding force applied to bend the folded edge structure shown in Figure 4b. Assuming the same net deflection for the structures with different edge lengths, $l$ and $L$, in Figure $4 \mathrm{a}, \mathrm{b}$, respectively $(L>l)$, and the same width $b$ and height $h$ for the two cases considered, the following relation is obtained:

a

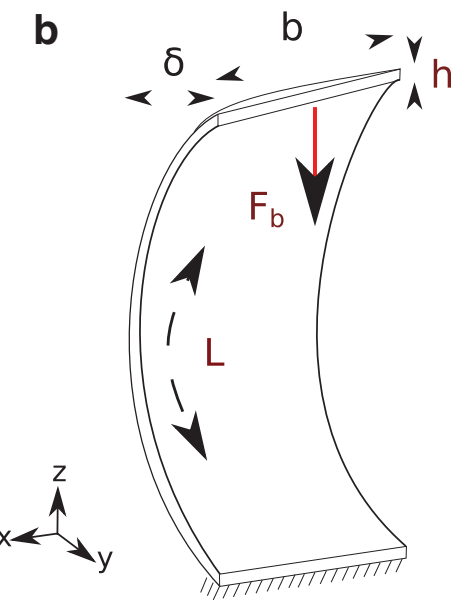

FIG. 4. Schematic to approximate the mechanical behavior of the paper folded vertex in the origami shell. The same width $b$ and thickness $h$ is used in both cases. A small length $l$ is used in (a) and a larger length $L$ is used in (b). It is concluded that the shell pattern with smaller fold tips, as shown in (a), produces a higher stiffness in bending direction, compared to the pattern shown in (b). Color images available online at www.liebertpub.com/soro

$$
\delta=\frac{4 F_{a}}{E} \frac{l^{3}}{b h^{3}} \frac{4 F_{b}}{E} \frac{L^{3}}{b h^{3}} .
$$

From Equation (2), the expression for the force $F_{a}$ is expressed in terms of the force $F_{b}$ as follows:

$$
F_{a}=F_{b} \frac{L^{3}}{l^{3}}
$$

This relation indicates that the paper shell pattern with smaller fold tips, as shown in Figure 3a, produces a higher stiffness in bending direction.

Compliance analysis. Figure 5 shows the net force needed to compress the shells in the plane $x z$ in direction $-x$ at different elongations. Besides a near-linear compliant behavior, the results show that the pattern that uses smaller fold paper tips, as seen in Figure 3a, presents a high stiffness. Based on the analysis above, the shell pattern shown in Figure $3 b$ was selected to carry out further analysis. The pattern is defined in terms of the parameter $\mathrm{C}$, which corresponds to the length of the folded paper tip, as shown in Figure 3. The number of folds selected for the final shell was set to five folds (corresponding to a total shell length of $10 \times \mathrm{C}$ ), as it allows the complete range of $0^{\circ}-90^{\circ}$ for bending.

\section{Origami cut pattern design}

The pattern of laser cut lines contributes largely to the performance of the final shell and is discussed below. The carbon dioxide laser machined folding lines provide repeatability 


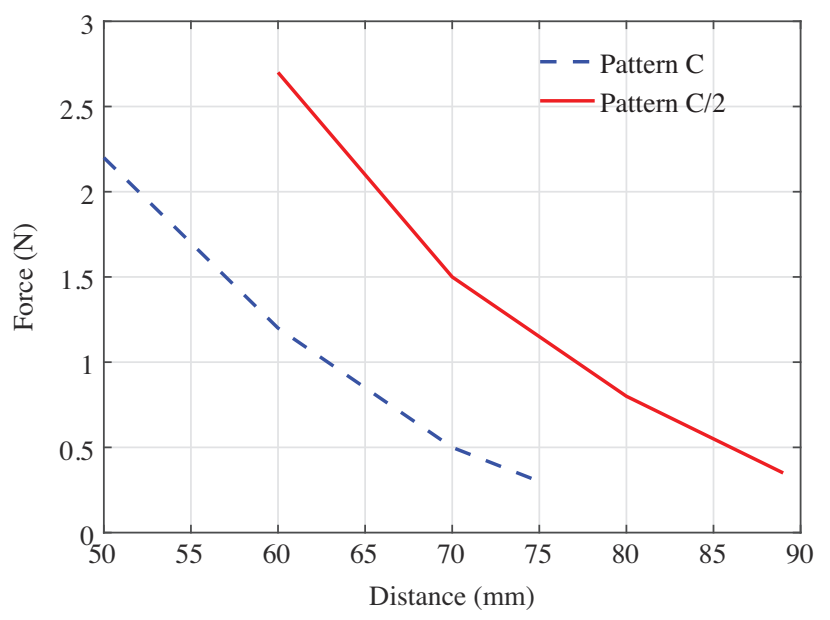

FIG. 5. Compressing force versus shell's length, for origami shell patterns shown previously in Figure 3. The paper thickness used for these tests corresponds to $300 \mathrm{~g} / \mathrm{m}^{2}$ area density. It is seen from the graph that the shell pattern that uses smaller folded paper tips (pattern $\mathrm{C} / 2$ ), as seen in Figure $3 \mathrm{a}$, presents a high stiffness compared to the shell pattern that uses twice as much folded tip length (pattern $C$ ). Color images available online at www.liebertpub.com/soro

across the folding process. Compliance and resilience test analysis for the different patterns produced on the shell is done to characterize its mechanical behavior and to select the most suitable pattern to be used in the final bending SPA module.

Compliance analysis. To determine the effect of the laser cut pattern on performance, sample papers with different cut patterns were tested. A paper with thickness corresponding to area density of $120 \mathrm{~g} / \mathrm{m}^{2}$ was used, and patterns with dashed dot and dashed lines were analyzed. Figure 6 shows four cut patterns assessed and the resulting net force needed to compress the sample. Each sample consisted of a rectangle, $80 \mathrm{~mm}$ in length and $30 \mathrm{~mm}$ in width. Every $20 \mathrm{~mm}$ of length was cut with the pattern, making a total of three lines across the sample. Once the sample is folded, it has the shape of a capital case sigma character $\Sigma$. The concave angle produced in the center fold versus the vertical compressing force is shown in Figure 6. As seen from the graph, the pattern with dashed dot and small gap is stiffer than the pattern with dashed line and long gap.

Resilience test. To ensure adequate compliance for the bending of the enclosed SPA, the dashed line pattern was selected for testing. Two different laser patterns were proposed as shown in Figure 7. The first pattern, shown in Figure 7a, does not include the convergence center of the paper folding, while the second pattern, shown in Figure 7b, includes the convergence center; the second pattern allows simplification of the folding of the shell structure and adds accuracy. To compare the strength of shell created using these two different patterns, a resilience test, consisting of compressing/decompressing the shell several times, was carried out with these patterns. The results show that the paper strength in the convergence centers of the paper folding was diminished proportionately with the number of times the shell was manipulated. The more accurately cut pattern,

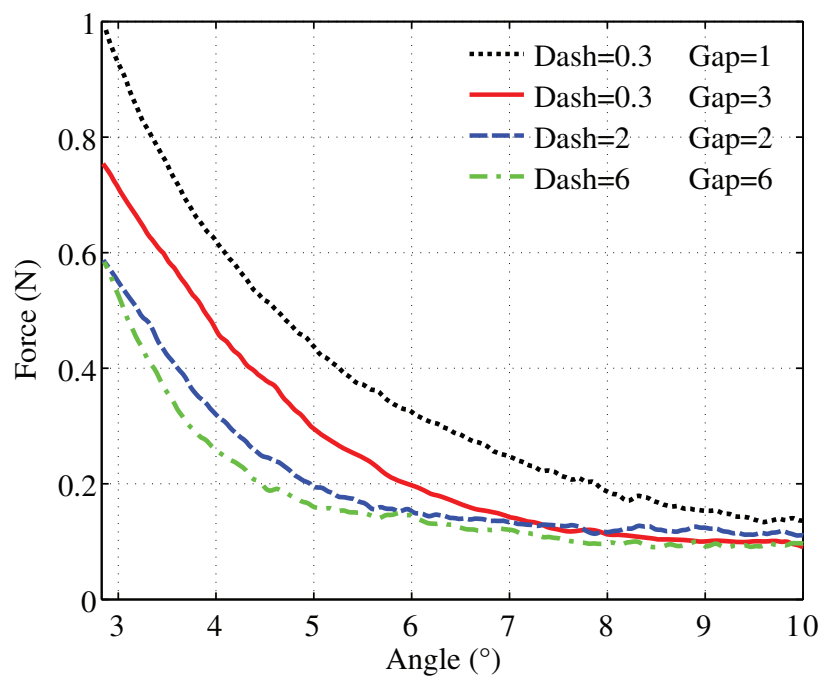

FIG. 6. Compressing force versus bending angle for samples with different shell cut patterns, to determine the effect of the laser cut pattern on module performance. The dashed cut and gap length values are in $\mathrm{mm}$. The paper thickness used corresponds to $120 \mathrm{~g} / \mathrm{m}^{2}$ area density. It is seen from the graph that the patterns with smaller dashes (dashed dots) and smaller gaps are stiffer than the patterns with longer dashes (dashed lines) and longer gaps. Color images available online at www.liebertpub.com/soro

including the convergence centers of the folds, as shown in Figure 7b, breaks after around 200 compression cycles, while the pattern without including the convergence centers of the folds (shown in Fig. 7a) proved to be more resilient to compression and manipulation. For this pattern, after around 1000 compressions, the paper strength was diminished but the shell still remains intact without holes.

\section{Selection of paper thickness}

Commercial laser printer paper was used here to test the effect of paper thickness, with area density ranging from 80 to $160 \mathrm{~g} / \mathrm{m}^{2}$. They correspond to thickness between $h=0.10$ and $0.34 \mathrm{~mm}$, respectively. Several A4 papers with area densities of $80,90,100,120$, and $160 \mathrm{~g} / \mathrm{m}^{2}$ were tested to investigate the folding edge compliance.

Deflection characteristic. As with the analysis carried out in the previous section to study the effect of different folded paper edge lengths on the resulting stiffness of the shell, a similar analysis is done to study the effect of varying paper thickness, using Equation (1). Figure 8 shows schematic diagrams illustrating the folded paper edges of the shell with two different paper thicknesses. As shown previously in Figure $4, F_{a}$ is the force applied to bend the folded edge structure shown in Figure $8 \mathrm{a}$ and $F_{b}$ is the corresponding force applied to bend the folded edge structure shown in Figure $8 \mathrm{~b}$. Assuming the same net deflection for the structures with different thickness $h$ and $H$ in Figure $8 \mathrm{a}$, b, respectively $(H>h)$, and the same width $b$ and length $L$ for the two cases considered, the following relation is obtained:

$$
\delta=\frac{4 F_{a}}{E} \frac{L^{3}}{b h^{3}}=\frac{4 F_{b}}{E} \frac{L^{3}}{b H^{3}} .
$$


FIG. 7. Origami shell patterns created with carbon dioxide laser cutter using $160 \mathrm{~g} / \mathrm{m}^{2}$ area density commercial inkjet paper. In the pattern shown in (a) the convergence center of folds is omitted. The pattern in (b) has the convergence center included in the cut. A zoomedin view of the origami pattern near the vertex region is shown below both the laser patterns after 1000 compressions in (a) and after 200 compressions in (b). The pattern shown in (b) tears up at the convergence centers, while the pattern in (a) remains intact and exhibits higher mechanical strength. Color images available online at

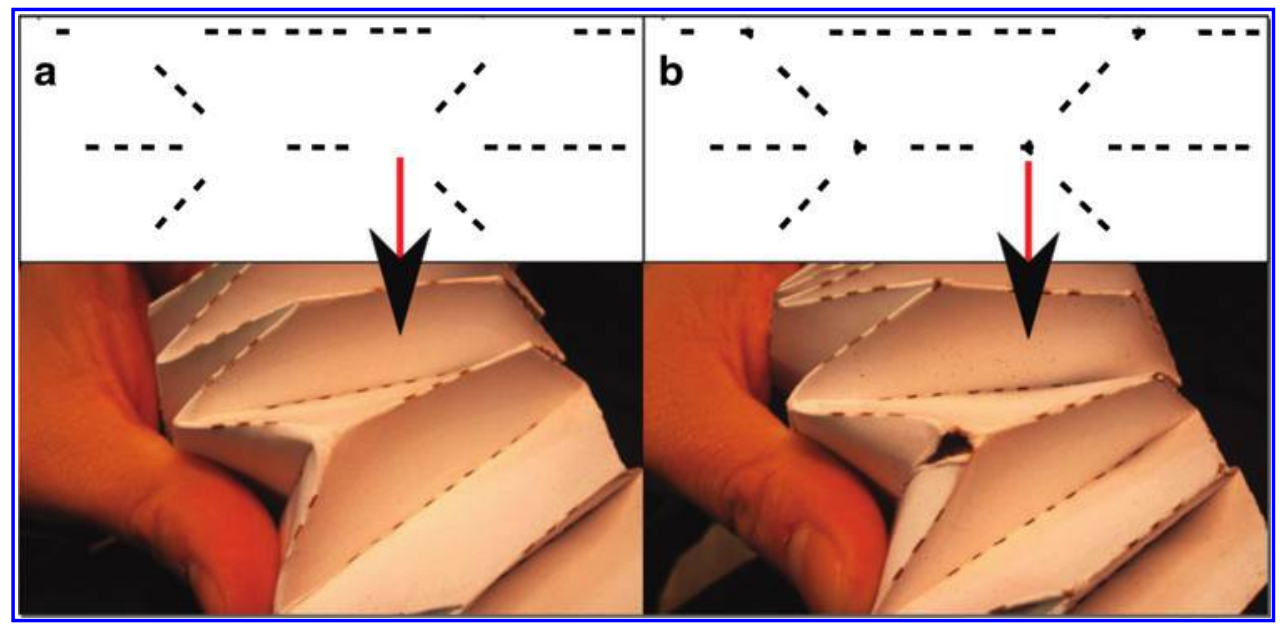
www.liebertpub.com/soro

From the above equation, the expression for $F_{b}$ is expressed in terms of $F_{a}$ as follows:

$$
F_{b}=F_{a} \frac{H^{3}}{h^{3}} .
$$

This relation implies that larger paper thickness increases the amount of force needed for the actuator to bend the folded paper tips and hence the entire shell, making it stiffer. The stiffness of shell is desired to allow the guided motion of the actuator while providing adequate robustness.

Compliance test. Longitudinally, the shell acts as a spring. Consequently, its blocked force behavior over the range of displacements can be measured to estimate its compliance at different operating points. In Figure 9, the experimental setup used for this purpose is shown. At the top, there is a motor with an encoder. When motor is actuated, a worm drive with an attached fixed plate is moved. At the bottom, there is a six-axis force/torque sensor (ATI Nano17), which obtains the contact force feedback. Fixed to the sensor, there is another plate. The shell is located between the two plates and is compressed while the motor is actuated and the worm drive moves down. The measure of the shell length

a

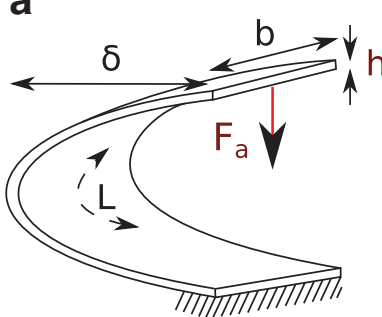

\section{b}

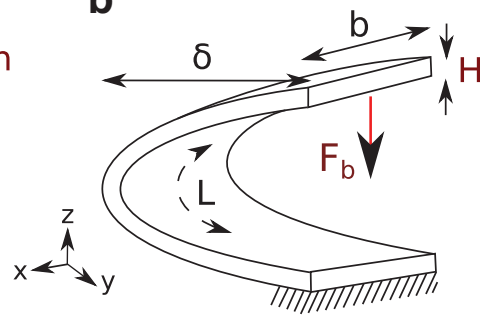

FIG. 8. Schematic to approximate the mechanical behavior of the paper folded vertex. The same width $b$ and length $L$ are used in both cases. The folded paper has a smaller thickness $h$ in (a) and a larger thickness $H$ in (b). It is concluded that larger paper thickness increases the amount of force needed for the actuator to bend the folded paper tips and hence the entire shell, making it stiffer. Color images available online at www.liebertpub.com/soro while it is compressed is obtained with the help of the encoder. The results for the shell compliance test are shown in Figure 10. Different paper thicknesses were tested, with area densities ranging from 80 to $160 \mathrm{~g} / \mathrm{m}^{2}$. For large paper densities between 120 and $160 \mathrm{~g} / \mathrm{m}^{2}$, and corresponding paper thicknesses, the paper shell offers a high resistance to bending.

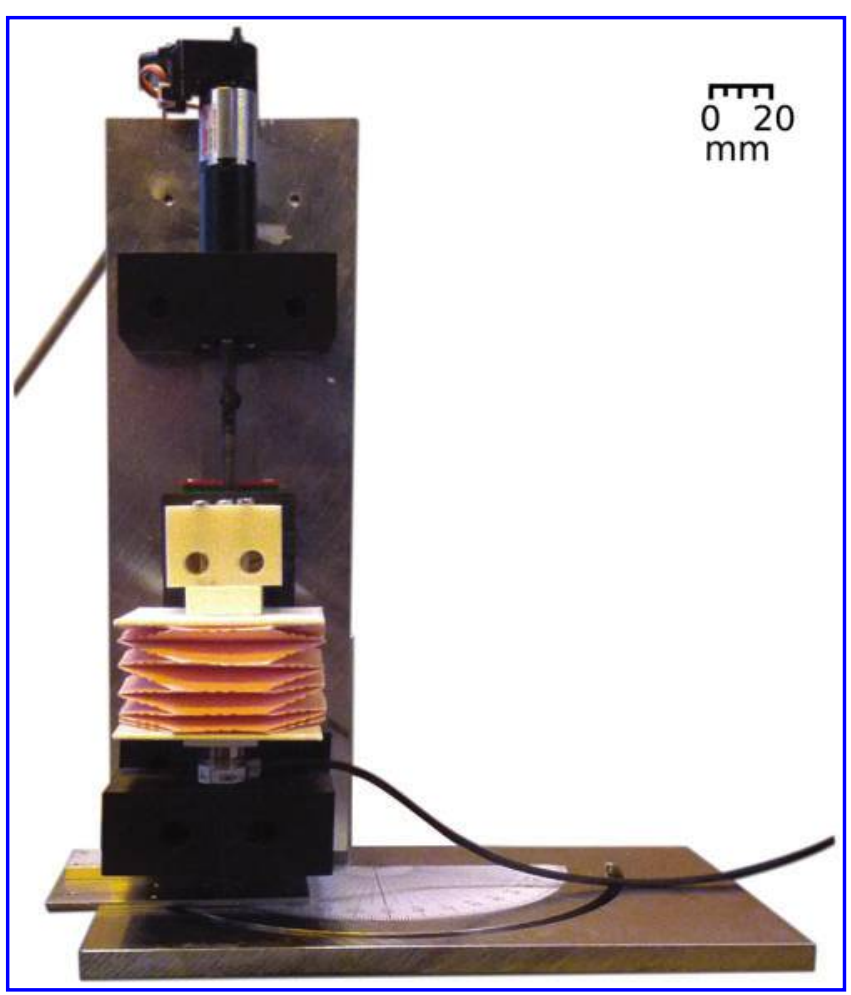

FIG. 9. Experimental setup used to test shell's compliance. At the top, there is a motor with an encoder. When motor is actuated, a worm drive with an attached fixed plate is moved. At the bottom, there is a six-axis force/torque sensor (ATI nano17), which obtains the contact force feedback. Fixed to the sensor, there is another plate. The shell is located between the two plates and is compressed while the motor is actuated and the worm drive moves down. Color images available online at www.liebertpub.com/soro 


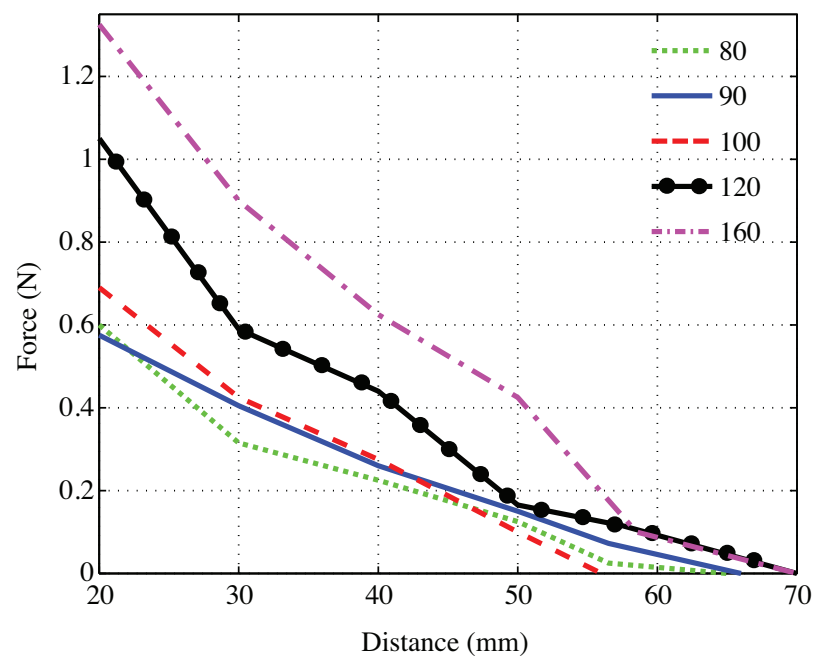

FIG. 10. Plot showing compression force versus shell's length for different shell paper's area densities, ranging between 80 and $160 \mathrm{~g} / \mathrm{m}^{2}$ and correspondingly thicknesses. For large paper densities between 120 and $160 \mathrm{~g} / \mathrm{m}^{2}$, and corresponding paper thicknesses, the paper shell offers a high resistance to bending. Color images available online at www.liebertpub.com/soro

\section{SPA Design}

The core actuating member in the bending module proposed here is the bending SPA. ${ }^{11,40}$ The SPA is fabricated using Ecoflex ${ }^{\circledR}$ 00-30 silicone rubber (from Smooth-On, Inc., with elastic modulus, $\mathrm{E}=69 \mathrm{kPa}$ ). To encase the SPA with dimensions as listed in Table 1 , the shell with dimensions (length/width/height) of $70 \mathrm{~mm}$ was chosen for further analysis. This SPA geometry was adapted from the SPA design used in Sun et al. ${ }^{11}$ and improvised for achieving the required motion profile in the present work. To achieve bidirectional bending, it is necessary to use two bending SPAs attached to each other, as shown in Figure 11. Despite the need for two air inlets, the use of a fabric as a common base for two silicone rubber modules results in a single SPA

Table 1. Bending Soft Pneumatic Actuator Design Parameters

\begin{tabular}{lc}
\hline Parameter & Value \\
\hline Air chamber size (width $\times$ height), mm & $2 \times 4$ \\
Air chamber length, mm & 8 \\
Number of chambers per side & 12 \\
Wall thickness, mm & 3 \\
Actuator height, one side, mm & 6.9 \\
Actuator height combining & 14 \\
$\quad$ two sides, including fabric, mm & \\
Actuator width, mm & 14 \\
Actuator length, mm & 71.6 \\
Material & Ecoflex ${ }^{\circledR} 00-30$, \\
& $\mathrm{E}=69 \mathrm{kPa}$ \\
Tube diameter, mm & 2 \\
Tube length, mm & 40 \\
Number of tube inlets & 2 \\
Limit pressure, $\mathrm{kPa}$ & 47 \\
Weight, g & 16.07 \\
\hline
\end{tabular}

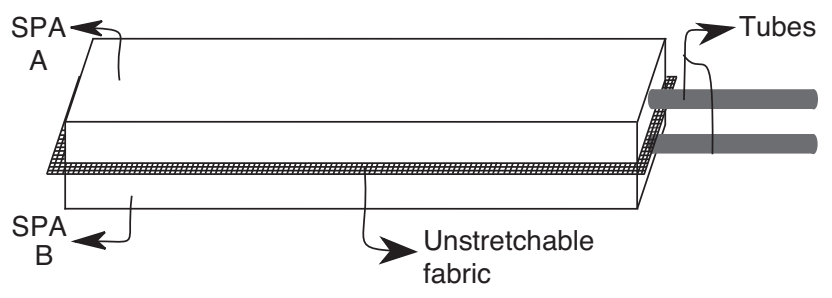

FIG. 11. Agonist/Antagonist structure of the bidirectional bending SPA. It consists of two bending SPAs made with silicone rubber, jointed at a common base made of unstretchable fabric. The piece of fabric in the middle of the construction constrains the length of the bidirectional actuator such that if one of the chambered sides inflates, a bending motion toward the opposite side is produced.

bending actuator unit with an agonist/antagonist behavior. The unstretchable fabric in the middle of the construction constrains the length of the bidirectional actuator such that if one of the chambered sides inflates, a bending motion toward the opposite side is produced. An example of the bidirectional SPA bending capabilities achieved with this unit is shown in Figure 12. The motion of the SPA without the shell is shown in Figure 12a, b, while that with the shell is shown in Figure 12c, d, at a similar level of bending motion. It is seen that symmetry is achieved in both the directions of bending, with as well as without the shell, when the same amount of pressure is applied through the primary air inlet while opening up the other air inlet to atmospheric pressure. At moderate to high values of input pressure, the shell does not over restrict the motion of the contained SPA and enables achievement of the desired motion profile similar to that of the actuator alone. In addition, by constraining excessive inflation of the contained SPA, the shell also allows for guided motion of the SPA at higher values of input pressure. A compendium of the design parameters used to create the bidirectional bending SPA is reported in Table 1. The integrated bidirectional bending module is shown in Figure 1 and has a total weight of $\mathrm{W}=45.33 \mathrm{~g}$, including the air tubes.

\section{Complete SPA-Origami Shell Module Assembly and Experimental Results}

Once the module with the shell and SPA actuator is assembled, several experiments are carried out to test its mechanical performance. These tests are aimed at characterizing the relationship between the input pressure and the resulting bending angle and the total torque exerted for the combined bending module.

\section{Bending angle}

To characterize the variation in bending angle with respect to the variation in input pressure, five actuators were used and each one was tested in both directions of motion. A step signal at different values of pressure was applied to each module. Video recordings of the sequences were taken until the module achieved a stable bending condition and the final bending angles were obtained from these. The average values of the resulting bending angles obtained as a function of applied pressure are plotted in Figure 13, for both the cases of the module with and without the shell, and in two directions 
FIG. 12. Bidirectional motion of the SPA without the shell, seen in (a, b), and with the shell, seen in $(\mathbf{c}, \mathbf{d})$, at a similar level of bending motion. Symmetry is achieved in both the directions of bending, when the same amount of pressure is applied through the primary air inlet, while opening up the other air inlet to atmospheric pressure. Color images available online at www.liebertpub.com/soro
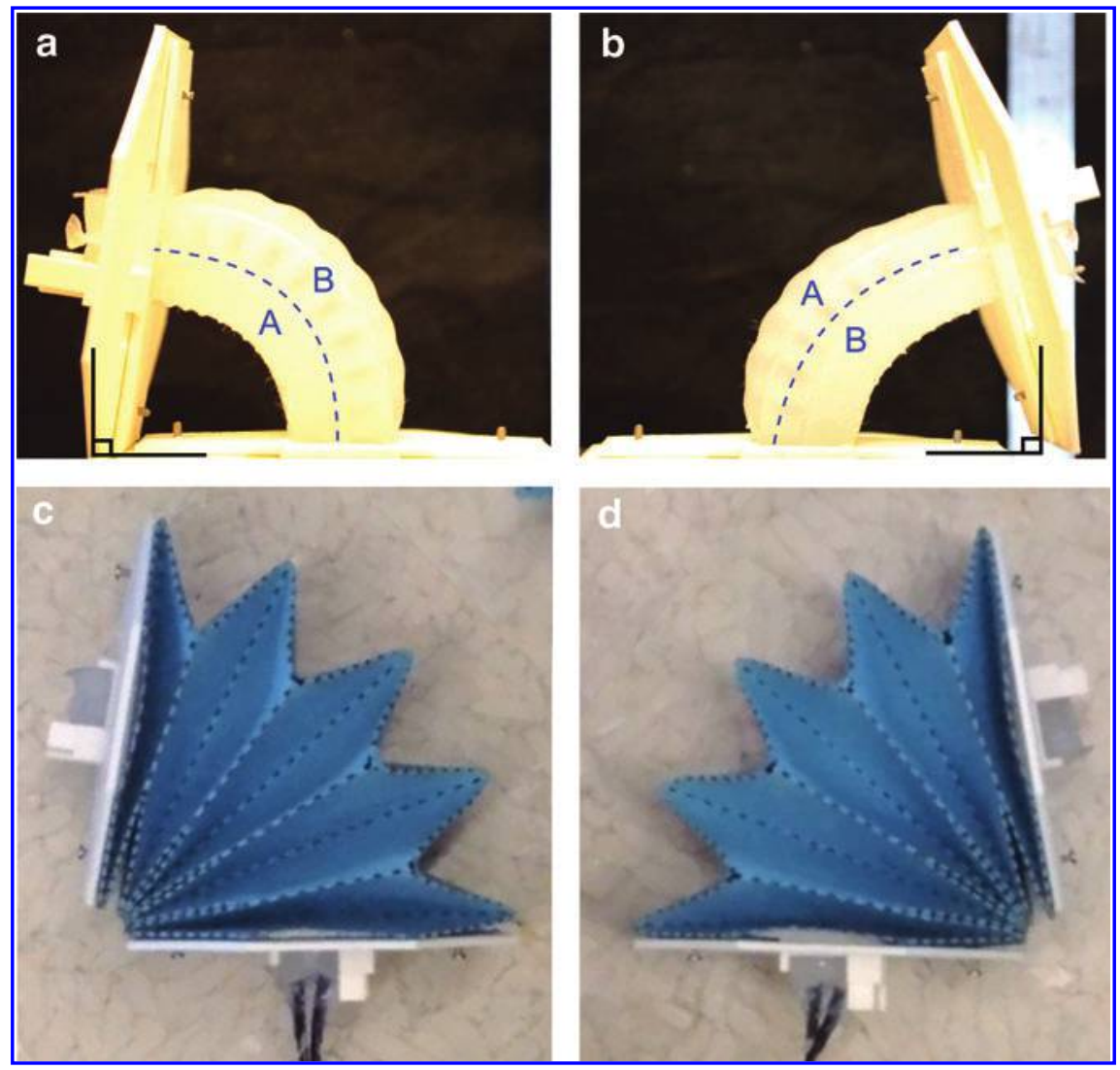

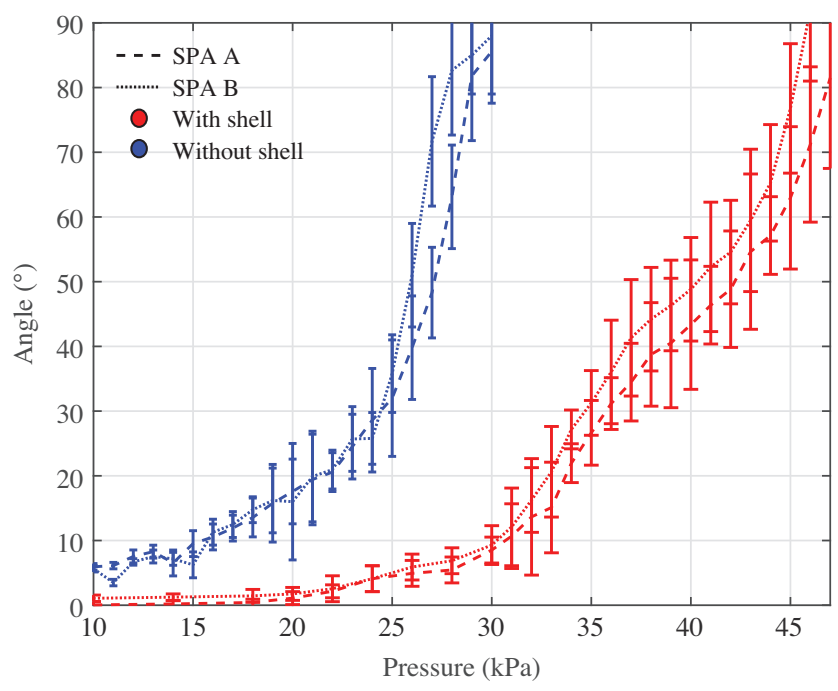

FIG. 13. The average values of the bending angles obtained as a function of applied pressure, for the bending module with and without the shell and in two different directions of motion governed by selective actuation of the soft actuators A and B. It is observed that although higher pressures are required to move the additional weight of the bending module with shell compared to the module without shell, the module with SPA alone can operate well only until lower pressures of around $30 \mathrm{kPa}$ before incurring mechanical damage. The bending module is capable of sustaining larger inflation pressures when the shell is attached. Color images available online at www.liebertpub.com/soro of motion. It is observed from the plot, although higher pressures are needed to move the additional weight of the bending module with the shell compared to the module without the shell, the module with the SPA alone can operate well only until lower pressures of around $30 \mathrm{kPa}$. At higher values of pressure, the module with SPA alone incurs damage and eventually mechanical rupture due to unconstrained excessive inflation. The bending module is capable of sustaining larger inflation pressures when the shell is attached, since the shell constrains the lateral motion of the actuator and provides guidance for actuator motion along the prescribed trajectory.

\section{Bending torque}

The experimental setup for measuring the torque with varying input pressure at a constant bending angle is shown in Figure 14. The setup consists of two plates that can be fixed to achieve a specific angle between them. One of the plates is attached to a fixed wall. The other plate is attached to a load cell with $10 \mathrm{~N}$ and $2 \mathrm{mV} / \mathrm{V}$ of sensitivity. The moving plate and the load cell can change their orientation and be fixed at a desired angle. The plate attached to the load cell is rigid with no deflection, ensuring a perpendicular force vector at the measuring point of the load cell. The load cell is set at a distance of $100 \mathrm{~mm}$ (independent of the angle tested) from the center of rotation to facilitate an easy reading of the torque exerted by the bending module.

To implement the experiments, a signal of variable pressure is set. The signal increments the pressure in the regulator every $3 \mathrm{~s}$ and by increments of $1 \mathrm{kPa}$. Plots for the 


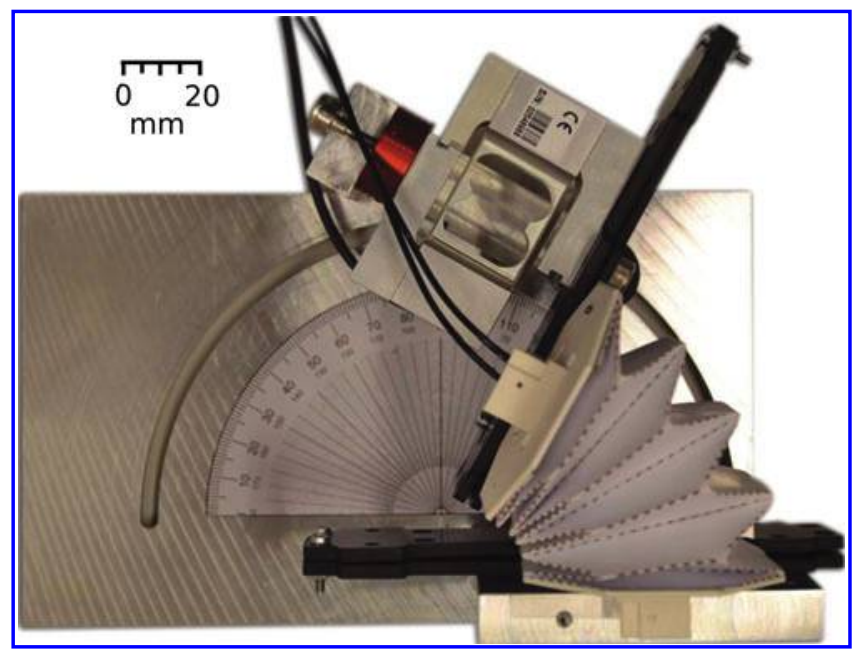

FIG. 14. Experimental setup for torque measurements. The setup consists of two plates that can be fixed to achieve a specific angle between them. One of the plates is attached to a fixed wall, while the other plate is attached to a load cell. The moving plate and the load cell can change their orientation and be fixed at a desired angle. The bending module is set up to achieve a bending angle of $60^{\circ}$. Color images available online at www.liebertpub.com/soro

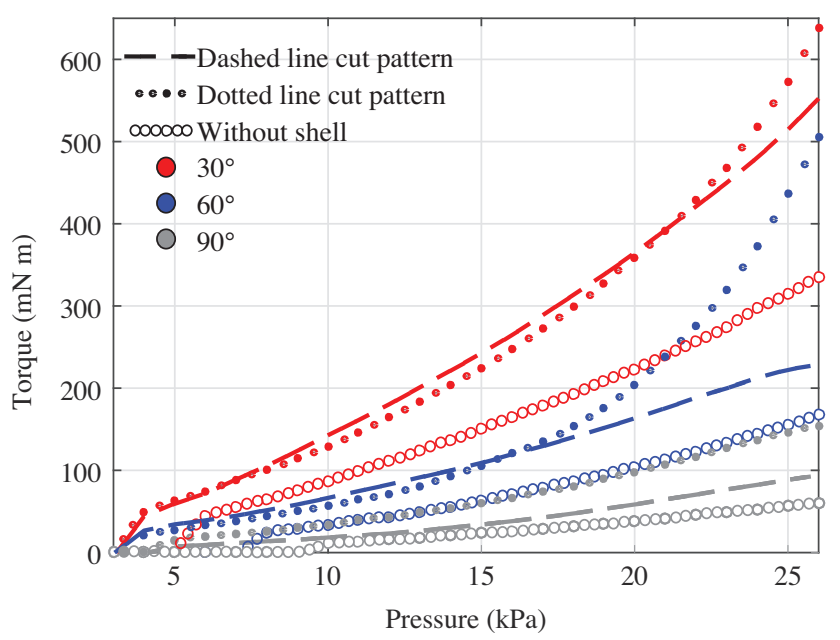

FIG. 15. Measurement of torque versus input pressure recorded at constant bending angles. The measurement is obtained for three different angles. The module was tested at each angle three times in different arrangements. The first and the second rounds of tests involve testing the SPA with the origami shell, using shells with dashed line cut pattern ( $2 \mathrm{~mm}$ dash, $2 \mathrm{~mm}$ gap) and dotted line cut pattern $(0.3 \mathrm{~mm}$ dot, $3 \mathrm{~mm}$ gap), respectively. The third measurement records the performance of the SPA under the same conditions without any shell reinforcement. The output torque for the bending module increases as the pressure increases. Larger torque values are obtained for lower bending angles. For each bending angle studied, the actuators encased within the origami shell deliver a significantly larger torque than the actuators without the shell, thereby enhancing the performance of the SPA considerably, especially at higher values of input pressure. Color images available online at www .liebertpub.com/soro measured torque versus pressure are shown for the actuator in Figure 15 , at angles of $30^{\circ}, 60,^{\circ}$ and $90^{\circ}$, for the cases of the actuator without the origami shell, the actuator with the shell with the dashed line cut pattern, and for the actuator with the shell with the dotted line cut pattern. The maximum pressure used is limited to $30 \mathrm{kPa}$ for these measurements. The output torque for the bending module increases as the pressure increases. Larger torque values are obtained for smaller bending angles.

For each bending angle studied, the actuators encased within the origami shell deliver a significantly larger torque than the actuators without the shell. This gap between the actuator performance with the origami shell reinforcement and without the shell increases with increase in input air pressure, leading to a largely improved mechanical performance obtained with the combined bending module at higher input operation pressures. In general, commonly encountered mechanical failure issues in SPAs occur at higher values of input pressure, since the actuator undergoes unconstrained large deformation in these situations. Thus, the progressively increasing improvement in SPA performance with increasing input pressure, obtained with the aid of the origami shell, is a highly desirable characteristic for such systems. This trend establishes the critical role played by the shell in enhanced performance obtained with the combined bending module, as opposed to the performance obtained with bending SPA alone. It is also observed that while at lower pressures, the dashed and dotted line cut patterns exhibit similar performance at higher pressures, the performance delivered with the dotted cut line pattern origami shell is superior to that obtained with the dashed cut line pattern, in terms of the maximum torque delivered. This is with the exception of the $90^{\circ}$ bending angle where the overlap is much less between the two designs. This is explained by the larger stiffness of the dotted cut pattern origami shell resulting from larger surface area coverage of the contained actuator in this case compared to the dashed line cut pattern origami shell.

\section{Conclusions}

The design and analysis of a novel soft bending module composed of a paper origami shell and a bidirectional bending SPA core, and the module's mechanical performance is presented in this work. The use of a shell surrounding the actuator helps to counteract inherent disadvantages suffered by the SPAs, such as limited control and precision in movement and vulnerability to rupture in their walls. Together with the shell and the SPA, the combined bending module is able to successfully achieve the desired motion and force profile. From the design perspective, an extensive and rigorous experimental and theoretical search is performed to determine the best design parameters. The effects of paper size, paper thickness, and cut patterns on the performance of the shell are studied. The bending module is tested for performance characteristics, in terms of the bending angle and the blocked torque, as a function of the applied input pressure. It is demonstrated that bending angles of upto $90^{\circ}$ and sufficiently large values of blocked torque are easily achievable within the working pressure range. It is also established that significantly higher (up to $233 \%$ increase at the maximum values of input pressure considered) values of blocked torque are delivered by the combined bending 
module comprising both the origami shell and the soft actuator, compared to the torque obtained with the actuator alone, demonstrating the capability of the module to deliver the desired performance not achievable with conventional SPAs alone.

\section{Acknowledgments}

This work was partially funded by Swiss National Center of Competence in Research (NCCR) in Robotics and by EPFL research funds.

\section{Author Disclosure Statement}

No competing financial interests exist.

\section{References}

1. Rus D, Tolley MT. Design, fabrication and control of soft robots. Nature 2015;521:467-475.

2. Kim S, Laschi C, Trimmer B. Soft robotics: A bioinspired evolution in robotics. Trends Biotechnol 2013;31:287-294.

3. Laschi C, Cianchetti M. Soft robotics: New perspectives for robot bodyware and control. Front Bioeng Biotechnol 2014; 2:3.

4. Trimmer B. Soft robots. Curr Biol 2013;23:R639-R641.

5. Onal C, Wood R, Rus D. An origami-inspired approach to worm robots. IEEE/ASME Trans Mech 2013;18:430-438.

6. Song YS, Sun Y, van den Brand R, von Zitzewitz J, Micera $\mathrm{S}$, Courtine $\mathrm{G}$, et al. Soft robot for gait rehabilitation of spinalized rodents. In 2013 IEEE/RSJ International Conference on Intelligent Robots and Systems (IROS), November 2013, pp. 971-976.

7. Holland DP, Park EJ, Polygerinos P, Bennett GJ, Walsh CJ. The soft robotics toolkit: Shared resources for research and design. Soft Robot 2014;1:224-230.

8. Tolley MT, Shepherd RF, Mosadegh B, Galloway KC, Wehner M, Karpelson M, et al. A resilient, untethered soft robot. Soft Robot 2014;1:213-223.

9. Ilievski F, Mazzeo AD, Shepherd RF, Chen X, Whitesides GM. Soft robotics for chemists. Angew Chem 2011;123: 1930-1935.

10. Shepherd RF, Ilievski F, Choi W, Morin SA, Stokes AA, Mazzeo AD, et al. Multigait soft robot. Proc Natl Acad Sci U S A 2011;108:20400-20403.

11. Sun Y, Song YS, Paik J. Characterization of silicone rubber based soft pneumatic actuators. In 2013 IEEE/RSJ International Conference on Intelligent Robots and Systems (IROS), November 2013, pp. 4446-4453.

12. Shepherd RF, Stokes AA, Nunes R, Whitesides GM. Soft machines that are resistant to puncture and that self seal. Adv Mater 2013;25:6709-6713.

13. Martinez RV, Glavan AC, Keplinger C, Oyetibo AI, Whitesides GM. Soft actuators and robots that are resistant to mechanical damage. Adv Funct Mater 2014;24:3003-3010.

14. Lipson H. Challenges and opportunities for design, simulation, and fabrication of soft robots. Soft Robot 2014;1: 21-27.

15. Elsayed Y, Vincensi A, Lekakou C, Geng T, Saaj C, Ranzani $\mathrm{T}$, et al. Finite element analysis and design optimization of a pneumatically actuating silicone module for robotic surgery applications. Soft Robot 2014;1:255-262.

16. Wakimoto S, Suzumori K, Ogura K. Miniature pneumatic curling rubber actuator generating bidirectional motion with one air-supply tube. Adv Robot 2011;25:1311-1330.
17. Kwok SW, Morin SA, Mosadegh B, So J-H, Shepherd RF, Martinez RV, et al. Magnetic assembly of soft robots with hard components. Adv Funct Mater 2014;24:2180-2187.

18. Wehner M, Tolley MT, Mengüç Y, Park Y-L, Mozeika A, Ding Y, et al. Pneumatic energy sources for autonomous and wearable soft robotics. Soft Robot 2014;1:263-274.

19. Marchese AD, Katzschmann RK, Rus D. A recipe for soft fluidic elastomer robots. Soft Robot 2015;2:7-25.

20. Marchese AD, Onal C, Rus D. Soft robot actuators using energy-efficient valves controlled by electropermanent magnets. In 2011 IEEE/RSJ International Conference on Intelligent Robots and Systems (IROS), September 2011, pp. 756-761.

21. Cianchetti M, Ranzani T, Gerboni G, Nanayakkara T, Althoefer K, Dasgupta P, et al. Soft robotics technologies to address shortcomings in today minimally invasive surgery: the STIFF-FLOP approach. Soft Robot 2014;1:122-131.

22. Polygerinos P, Wang Z, Galloway KC, Wood RJ, Walsh CJ. Soft robotic glove for combined assistance and at-home rehabilitation. Rob Auton Syst 2014;73:135-143.

23. Polygerinos P, Lyne S, Wang Z, Nicolini LF, Mosadegh B, Whitesides GM, et al. Towards a soft pneumatic glove for hand rehabilitation. In 2013 IEEE/RSJ International Conference on Intelligent Robots and Systems (IROS), IEEE, 2013, pp. 1512-1517.

24. Tondu B. Modelling of the McKibben artificial muscle: a review. J Intell Mater Syst Struct 2012;23:225-253.

25. Tondu B, Lopez P. Modeling and control of McKibben artificial muscle robot actuators. Control Systems IEEE 2000;20:15-38.

26. Roche ET, Wohlfarth R, Overvelde JTB, Vasilyev NV, Pigula FA, Mooney DJ, et al. A bioinspired soft actuated material. Adv Mater 2014;26:1200-1206.

27. Connolly F, Polygerinos P, Walsh CJ, Bertoldi K. Mechanical programming of soft actuators by varying fiber angle. Soft Robot 2015;2:26-32.

28. Polygerinos P, Wang Z, Overvelde JTB, Galloway KC, Wood RJ, Bertoldi K, et al. Modeling of soft fiberreinforced bending actuators. IEEE Trans Robot 2015;31: 778-789.

29. Memarian M, Gorbet R, Kulic D. Modeling and experimental analysis of a novel design for soft pneumatic artificial muscles. In 2015 IEEE/RSJ International Conference on Intelligent Robots and Systems (IROS), IEEE, 2015, pp. 1718-1724.

30. Yoshimura Y. On the mechanism of buckling of a circular cylindrical shell under axial compression. NACA-TM1390, 1951.

31. Chen Y, Peng R, You Z. Origami of thick panels. Science 2015;349:396-400.

32. Filipov ET, Tachi T, Paulino GH. Origami tubes assembled into stiff, yet reconfigurable structures and metamaterials. Proc Natl Acad Sci U S A 2015;112:12321-12326.

33. Onal C, Wood R, Rus D. Towards printable robotics: Origami-inspired planar fabrication of three-dimensional mechanisms. In 2011 IEEE International Conference on Robotics and Automation (ICRA), May 2011, pp. 4608-4613.

34. Lee D-Y, Jung G-P, Sin M-K, Ahn S-H, Cho K-J. Deformable wheel robot based on origami structure. In 2013 IEEE International Conference on Robotics and Automation (ICRA), May 2013, pp. 5612-5617.

35. Peraza-Hernandez EA, Hartl DJ, Malak R, Jr, Lagoudas DC. Origami-inspired active structures: A synthesis and review. Smart Mater Struct 2014;23:094001. 
36. Paez L, Granados M, Melo K. Conceptual design of a modular snake origami robot. In 2013 IEEE International Symposium on Safety, Security, and Rescue Robotics (SSRR), October 2013, pp. 1-2.

37. Buri H, Weinand Y. ORIGAMI-Folded plate structures, architecture. In 10th World Conference on Timber Engineering, 2008.

38. Stavric M, Wiltsche A. Investigations on quadrilateral patterns for rigid folding structures-Folding strategies-Rigid and curved folding. In Open Systems: Proceedings of the 18th International Conference on Computer-Aided Architectural Design Research in Asia, 2013, p. 232.

39. Min CC, Suzuki H. Geometrical Properties of Paper Spring (Manufacturing Systems and Technologies for the New Frontier, 41st CIRP Conference on Manufacturing Systems, May 26-28, 2008, Tokyo, Japan) Mitsuishi M, Ueda K, Kimura F (Eds). Springer, 2008, pp. 159-162.
40. Moseley P, Florez J, Sonar H, Agarwal G, Curtin W, Paik J. Modeling, design, and development of soft pneumatic actuators with finite element method. Adv Eng Mater 2016;8: 978-988.

41. Howell LL. Compliant Mechanisms. John Wiley \& Sons, 2001.

Address correspondence to: Jamie Paik

Reconfigurable Robotics Lab Swiss Federal Institute of Technology (EPFL) MED 11326 (Batiment MED) Station 9 CH-1015 Lausanne Switzerland

E-mail: jamie.paik@epfl.ch 